\title{
Near-Field Microwave Sensor Composed of 3D Printed Antennas and Lenses
}

\author{
Sebastian G. Wirth* and Ivor L. Morrow* \\ ${ }^{*}$ Cranfield University, Laboratory of Electromagnetic Systems Engineering, \\ Shrivenham Campus, Oxfordshire, SN6 8LA, UK. \\ Email: sebastian.wirth@cranfield.ac.uk
}

\begin{abstract}
A novel near-field microwave imaging system was designed and fabricated using the three-dimensional (3D) printing technique to manufacture $\mathrm{X}$-band Pyramidal horn antennas and planar graded-index (GRIN) lenses. The flat lens focusing profile is synthesised by varying the refractive index radially in incremental steps that adjust the air-dielectric mixture. The lens is designed for direct attachment to the antenna aperture and transforms spherical waves emanating from antenna phase center into plane radiating waves. Measurement results show the antenna lens system input impedance is $\leq-10 \mathrm{~dB}$, radiation pattern gain is between 10-16 $\mathrm{dBi}$ over the 8-12 $\mathrm{GHz}$ frequency band and when arrayed for polarimetry sensing has a polarisation cross-talk of $\leq-35 \mathrm{~dB}$. The antenna lens system is suitable for ground penetrating radar applications.
\end{abstract}

Keywords: - 3D print manufacture, antenna, GRIN lens, polarimetry, microwave imaging.

\section{INTRODUCTION}

There is an ever increasing interest for next generation ground penetrating radar (GPR) antennas to be lighter and cheaper while performing at least as well as, or better than, conventional antenna types. Moreover, GPR antennas for humanitarian demining applications are required to operate in the near or quasi-near field of the terrain being surveyed. This paper describes the use of additive 3D print manufacture to design practical microwave antennas and lens components which exploit the low cost and light weight advantages of the technique and permit bespoke design features that would otherwise be difficult or expensive to procure commercially.

The 3D printing of microwave components [1] and lens technology [2] has recently been the subject of much research interest. Usually the lens is located some distance in front of the antenna at the near-field/far-field boundary. A variety of design approaches and 3D printing techniques have been recently reported. Hyperbolic profile lenses have been transformed to equivalent flat lens designs. The refractive index may be made to vary by fabricating the lenses as a series of subwavelength unit cell with tailored dielectric value. The range of dielectric values obtained by this 3D printing technique can introduce constraints on lens thickness. Recent efforts [3], [4] have investigated $3 \mathrm{D}$ printing metal particles and dielectrics. These artificial dielectrics are reported to have larger dielectric value permitting construction of thinner lenses. Alternatively, metamaterial surfaces based on slots cut in cascaded dielectrics have been proposed [5] at millimeter wave frequencies. Here 978-1-5386-5204-6/18/\$31.00 (C)2018IEEE the authors consider insertion of a constant thickness lens into the antenna aperture. This rapidly converts near-field spherical wavefronts emerging from the antenna phase center into planar phase fronts with enhanced directivity in the desired direction.

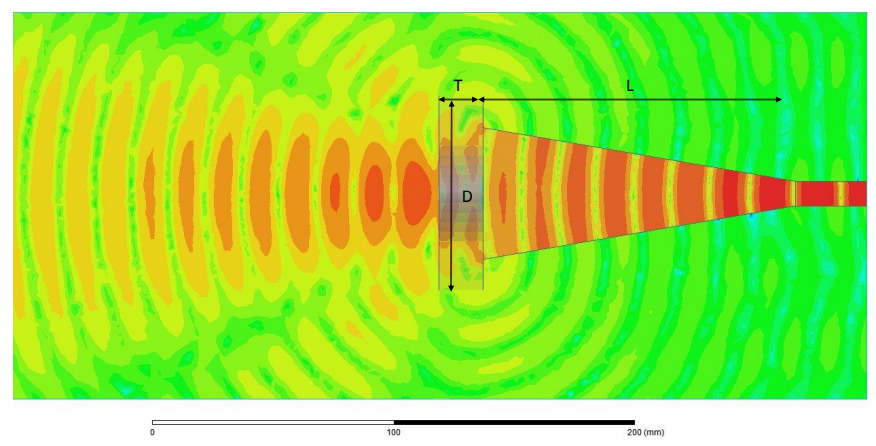

Fig. 1: Simulated electric field of the near-field GRIN lens at $10 \mathrm{GHz}$, fed by a rectangular X-band pyramidal horn. The lens is attached to the horn aperture and focused at the antenna phase center: $\mathrm{D}=100 \mathrm{~mm}, \mathrm{~T}=50 \mathrm{~mm}, \mathrm{~L}=142 \mathrm{~mm}$, WR90 $(10.16 \times 22.86) \mathrm{mm}$.

This paper is organised as follows; Section II describes the novel near-field lens design and 3D printing technique. Fig 1 illustrates a full-wave simulation of the electric field emerging from the lens integrated with an X-band Pyramidal horn antenna computed using the commercial software HFSS [6]. The horn antenna is also $3 \mathrm{D}$ printed and electroplated with a 40 micon thick copper coating. The horn, although not the subject of this paper, was designed according to standard pyramidal horn equations [7] to provide an optimum gain of $16.3 \mathrm{~dB}$ at $10 \mathrm{GHz}$.

HFSS simulation results for radiation patterns at 8,10 , and $12 \mathrm{GHz}$ frequencies determined the antenna phase center was located $50 \pm 1.0 \mathrm{~mm}$ inside the antenna aperture. The radiation pattern amplitude and phase were measured in an anechoic chamber and results confirmed that experimentally the phase center was located between $49-51 \mathrm{~mm}$ behind the horn aperture.

Section III shows some of the measured and simulated results for the horn antenna with the lens attached. In Section IV four near-field horn antennas are assembled into a polarimetry array and polarimetry cross-talk levels characterised. 
The polarimetry cross-talk results are critically evaluated and some conclusions drawn in Section V.

\section{Flat LENS DESIGN}

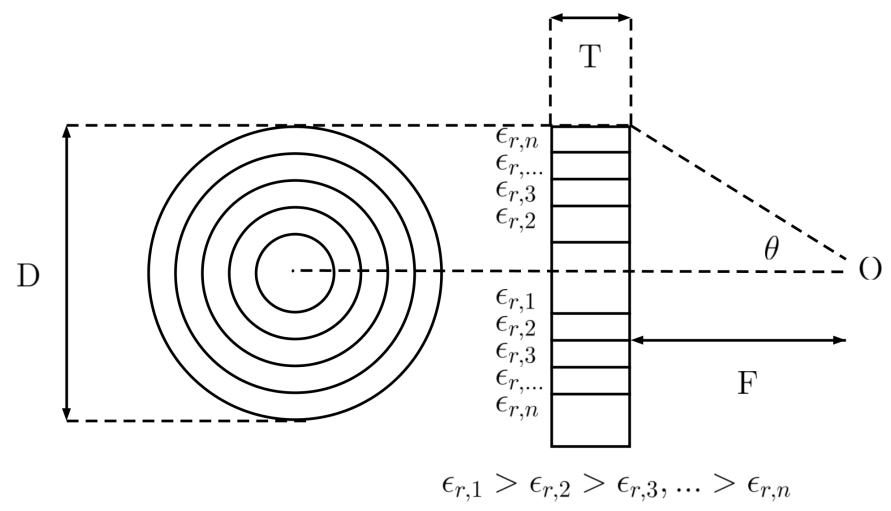

(a) front view

(b) side view

Fig. 2: Sketch showing the constant thickness lens composed of annular ring of decreasing dielectric constant material.

Fig. 2 shows a constant thickness, $T$, dielectric lens ${ }^{\dagger}$. composed of variable refractive index medium $\left(n=\sqrt{\epsilon_{r}}\right)$ and with focal point $F$ located on its axis of symmetry. The refractive index decreases linearly from a maximum at the centre of the lens to a value of 1.0 at the perimeter. One feasible $3 \mathrm{D}$ printing technique that would approximate this variation is incremental stepping of the refractive index. This permits fabrication as a series of annular dielectric rings of increasing diameter and decreasing permittivity towards the lens perimeter. The relationship between the radially varying $\epsilon_{r}$ and angle of incidence $\theta$ can be shown to be [8],

$$
\begin{array}{r}
T\left(\epsilon_{r}-\frac{2}{3} \sin ^{2}(\theta)\right)=\sqrt{\epsilon_{r}-\sin ^{2}(\theta)} \times \\
{\left[\sqrt{\epsilon_{m}} T-(\sec (\theta)-1)\right] .}
\end{array}
$$

where $T$ is the lens thickness, $\epsilon_{m}$ the maximum permittivity of the lens at the centre and $\epsilon_{r}$ is the relative permittivity of a particular annular ring at $\theta$ degrees to the focal point. Eqn. 1 is the basic design equation for the planar graded refractive index (GRIN) lens and is solved by the root bisection method in Table I to determine discrete values of $T$ for $\theta$. An initial estimation of the lens proportions may be made knowing the maximum value of $\theta=55^{\circ}$ is determined by the diameter of the lens and the focal length is fixed at $F=50 \mathrm{~mm}$. A fabrication constraint is also imposed on the minimum value of $\epsilon_{r}$ which can be printed and maintain the lenses structural strength. A feasible approach is to synthesis "artificial" dielectric values between 2.7- 1.3. One practical method is to exploit dielectric mixing principles and introduce shaped air voids into the base material. The Maxwell-Garnett,

\footnotetext{
${ }^{\dagger}$ generated by rotation about the principal axis
}

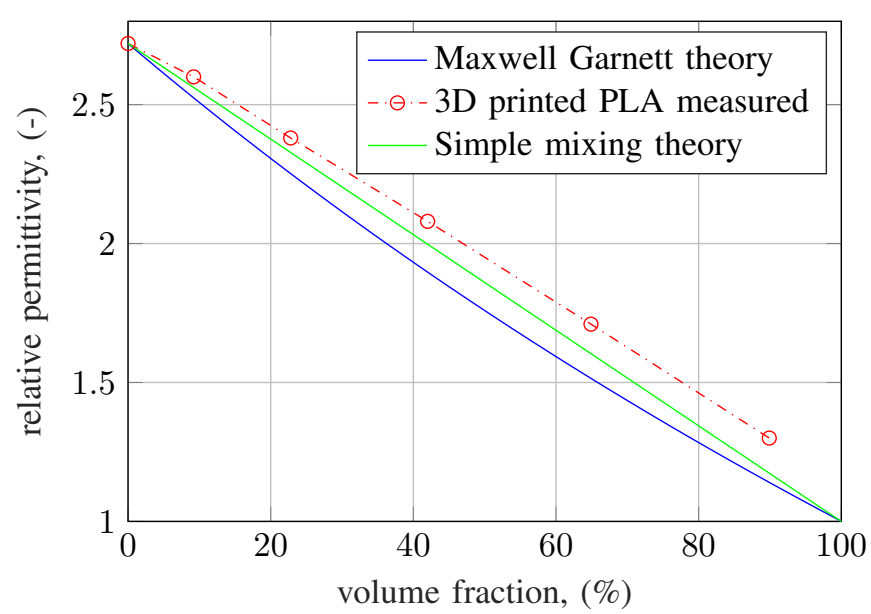

Fig. 3: Comparison of effective permittivity model prediction and measured permittivity for PLA and air over a range of volume fractions.

with Rayleigh approximation may be solved to determine the effective dielectric constant of the mixture [9] as,

$$
\epsilon_{e f f}=\epsilon_{m} \frac{2 \delta_{i}\left(\epsilon_{i}-\epsilon_{m}\right)+\epsilon_{i}+2 \epsilon_{m}}{2 \epsilon_{m}+\epsilon_{i}+\delta_{i}\left(\epsilon_{m}-\epsilon_{i}\right)} .
$$

The 3D printer base medium, is Poly Lactic Acid or PLA with permittivity $\epsilon_{m}=2.72$ and the air inclusion permittivity $\epsilon_{i}=1.0004$, where $\delta_{i}$ is the volume fraction of the inclusion. Fig 3 illustrates the relationship for effective permittivity for air inclusion volume fractions using; (i) Eqn. (2), (ii) measurement of some 3D printed PLA samples (published in [2]) and (iii) a simple linear volume mixing relationship. Clearly, the 3D printed and predicted effective permittivity values follow a nearly linear relationship. Equation (1) was solved subject to the constraints imposed on practical realisation of effective dielectric value and a fixed focus length. The results are summarised in Table I. The ideal lens thickness is between $47-51 \mathrm{~mm}$ and a trade-off value of $50 \mathrm{~mm}$ thickness was chosen.

TABLE I: Summary design data on the GRIN lens. Tabulated values of lens thickness versus concentric ring radius, effective dielectric constant and PLA/air volume fractions for the seven concentric rings (the ${ }^{*}$ value indicates a suspect $T$ value).

\begin{tabular}{llllll} 
Ring No. & $\mathrm{R}(\mathrm{mm})$ & $\theta(\mathrm{degs})$ & $\mathrm{T}(\mathrm{mm})$ & $\epsilon_{\text {eff }}$ & $\delta_{i} \%$ \\
\hline 1 & 10 & 5.0 & 49.69 & 2.72 & 100 \\
2 & 20 & 15.0 & 48.49 & 2.60 & 90.8 \\
3 & 30 & 25.0 & 45.96 & 2.38 & 77.1 \\
4 & 40 & 35.0 & 45.02 & 2.08 & 58.0 \\
5 & 50 & 45.0 & $43.70^{*}$ & 1.71 & 35.1 \\
6 & 60 & 55.0 & 47.85 & 1.30 & 10.1 \\
7 & 70 & 65.0 & 47.31 & 1.20 & 9.89 \\
\hline
\end{tabular}

The lens is comprised of seven concentric rings, each with a annular width of $10 \mathrm{~mm}$. Two approaches were taken to 3D printing the dielectric lens; the first was to 3D print the lens as a single whole piece and the second involved $3 \mathrm{D}$ printing each annulus separately and carefully push fitting together to 
form one whole lens. The latter approach was adopted since it allowed flexibility in modifying individual rings without reprinting the whole structure. The HFSS model of the solid lens was translated to the SolidWorks file format. The CAD file was then imported to Slic3r as an .stl file and densities assigned according to Table I. The annular rings were 3D printed using an Ultimaker 3 Extended printer with a 0.4 mm extrusion nozzle. The completed 3D printed GRIN lens is shown in Fig 4.

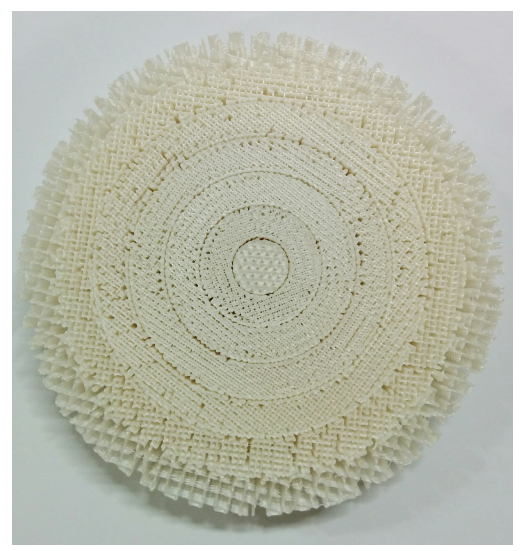

Fig. 4: Three-dimensional printed flat GRIN lens.

\section{RESUlts OF ANTENNA AND LENS INTEGRATION}

Simulation models for the X-band Pyramidal horn and GRIN lens were developed using HFSS. The lens was modelled as seven solid annular rings of homogeneous dielectric constant value according to Table I and assuming negligible loss tangent $\tan \delta=0.008$. Fig. 5 shows the reflection coefficient for the 3D printed horn antenna with and without the lens attached to the antenna aperture. Agreement between simulation and measurement is good. It is observed that the near-field lens has not affected the impedance match and appears to have improved it at higher frequencies. This implies that the introduction of any impedance matching layer is not required. The antenna radiation patterns were measured in an anechoic chamber and the gain calculated ( [7], p. 95). Fig 6 shows the measured and simulated gain are in good agreement, the near-field lens has introduced a perturbation in gain but this has not reduced the gain-bandwidth response.

\section{NEAR-FIELD SENSOR CHARACTERISATION}

An X-band full polarimetry SAR sensor was assembled to enable a GPR polarimetry system. The full polarimetry horn antenna array is shown in Fig. 7 with antennas orientated to provide full polarimetry and secured into a 3D printed support. The array consists of four standard gain horn antennas, two are 3D printed, the other two are commercial Narda horn antennas with similar performance. The horn antenna provide a cosine tapered illumination at the antenna aperture and the lens when placed centrally on the horn aperture has its focal point at the antenna phase centre and the beam is directed along the lens axis. The circular lenses are then trimmed to fit, and bonded to,

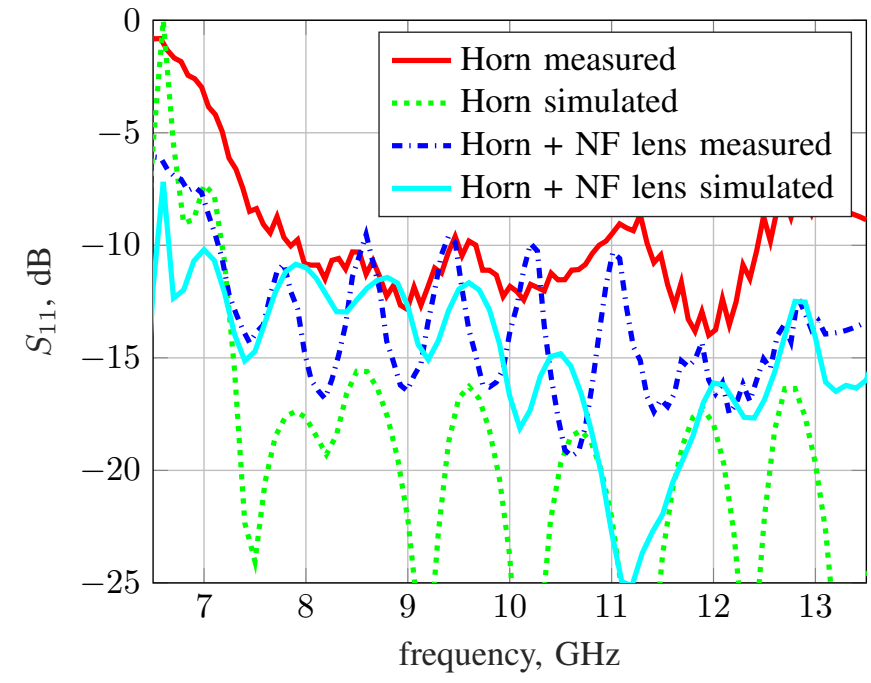

Fig. 5: Measured and simulated reflection coefficient of 3D printed horn antenna with and without near-field lens.

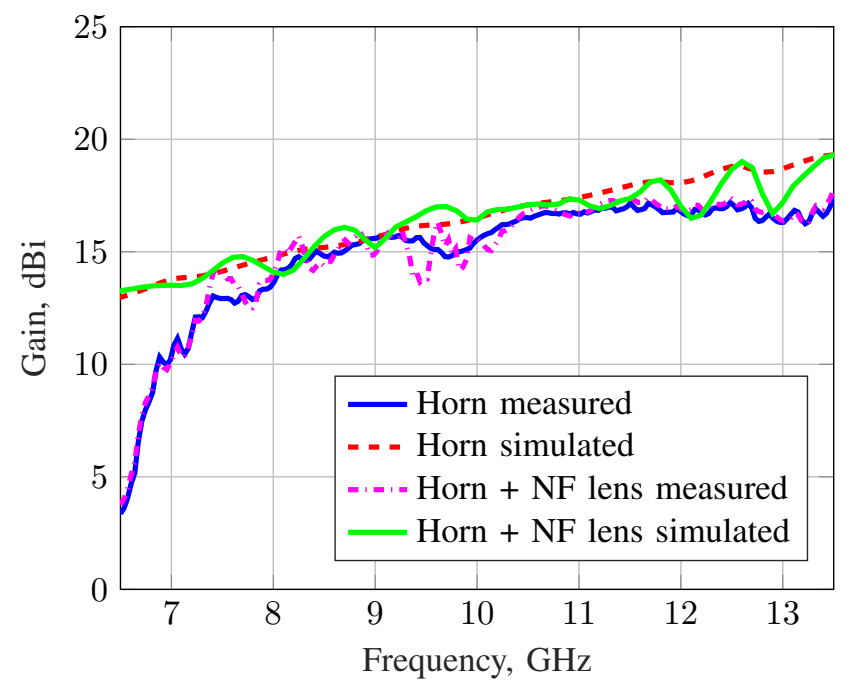

Fig. 6: Measured and simulated gain of 3D printed horn antenna with and without near-field lens.

the rectangular radiating apertures. The polarisation switch is a conventional PIN diode switch matrix able to switch $30 \mathrm{dBm}$ peak power from the transmitter while exhibiting low loss $(0.3$ $\mathrm{dB}$ ) and high isolation (more than $50 \mathrm{~dB}$ ). Thus system crosstalk (between polarisations) is dominated by antenna crosstalk. Fig. 8 demonstrates the measured $S_{11}$ response for all the antennas in the array are well matched. Figs. 9 and 10 show the measured co-polar mutual coupling in $\mathrm{E}-$ and $\mathrm{H}-$ planes of the polarimetry array are less than $-35 \mathrm{~dB}$ and the cross-polar mutual coupling in E- and H-planes is less than $-60 \mathrm{~dB}$.

\section{CONCLUSIONS}

This paper has described the 3D design and fabrication of a low-cost, light-weight planar microwave lens tailored for 


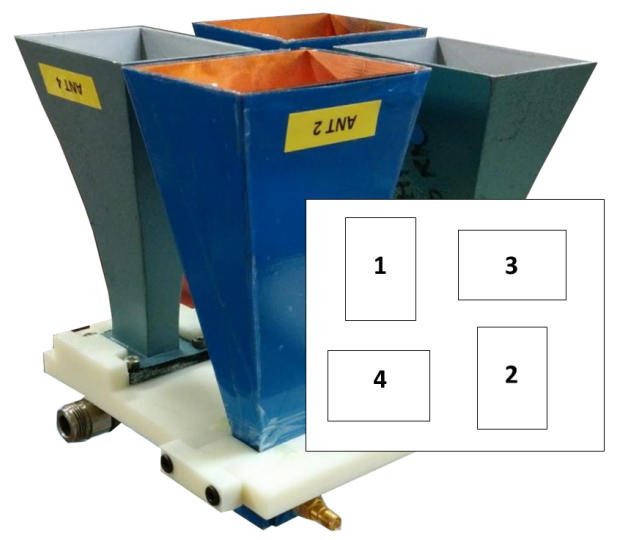

Fig. 7: Polarmetric array incorporating two 3D printed horn antennas and two commercial horns; lenses are not shown for clarity (the inset depicts the orientation of the horn apertures).

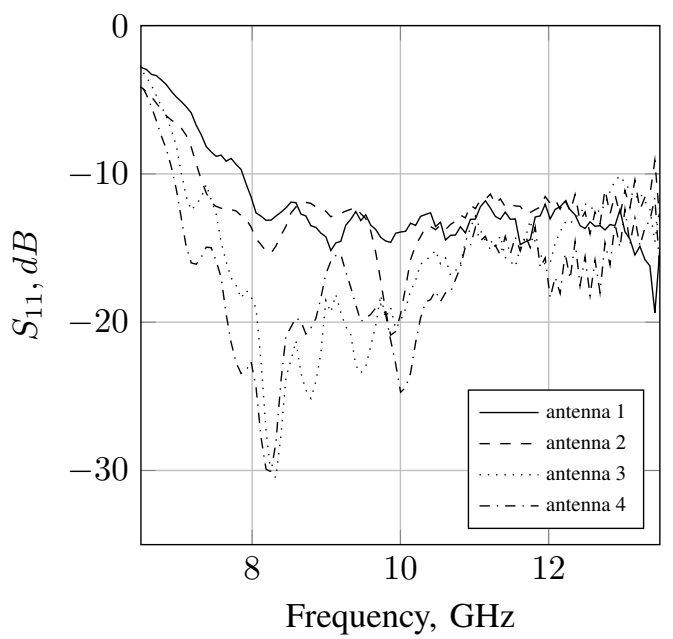

Fig. 8: Measured $S_{11}$ reflection coefficient for individual antennas in the polarmetric array (see Fig. 7 inset).

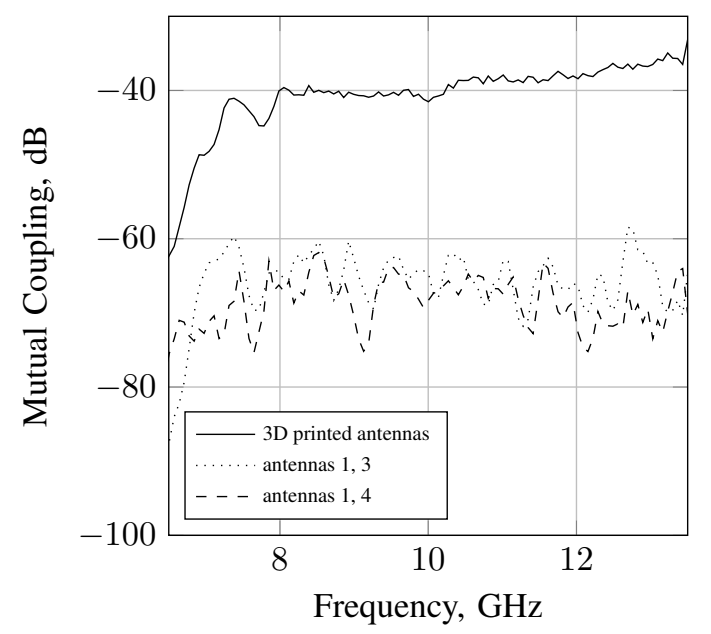

Fig. 9: Co- and cross-polar mutual antenna coupling in E-plane (see Fig. 7 inset).

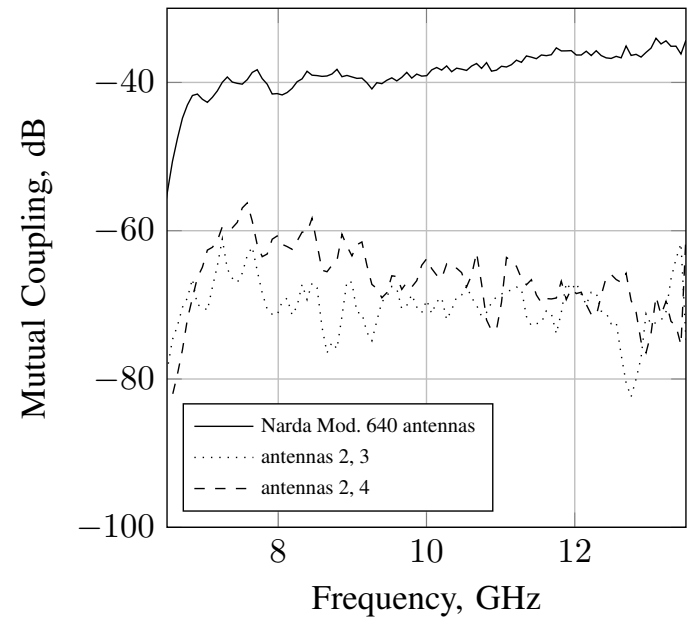

Fig. 10: Co- and cross-polar mutual antenna coupling in $\mathrm{H}-$ plane (see Fig. 7 inset).

near-field antenna systems. The horn antenna provide a cosine tapered illumination at the antenna aperture and the lens when placed centrally on the horn aperture has its focal point at the antenna phase centre. Thus transforming the spherical wave front emanating from the antenna phase center to a planar phase front and the beam is directed along the lens axis. The antenna input impedance and gain performance with the lens attached demonstrated good agreement between simulation and theory. The lensed antennas were arranged in a polarimetry array and measurements indicated the antenna cross-talk levels are below $-35 \mathrm{~dB}$. The low cross-talk levels in the polarimetry array are highly beneficial for GPR applications.

\section{ACKNOWLEDGMENT}

The authors thank the Find A Better Way charity for their support of this research under the DETERMINE programme (grant number 2015/001D).

\section{REFERENCES}

[1] K. V. Hoel, S. Kristoffersen, J. Moen, K. G. Kjelgrd, and T. S. Lande, "Broadband antenna design using different $3 \mathrm{~d}$ printing technologies and metallization processes," in 2016 10th European Conference on Antennas and Propagation (EuCAP), April 2016, pp. 1-5.

[2] S. Zhang, R. K. Arya, S. Pandey, Y. Vardaxoglou, W. Whittow, and R. Mittra, "3d-printed planar graded index lenses," IET Microwaves Antennas Propagation, vol. 10, no. 13, pp. 1411-1419, 2016.

[3] S. Zhang, W. Whittow, and J. . C. Vardaxoglou, "Additively manufactured artificial materials with metallic meta-atoms," IET Microwaves, Antennas Propagation, vol. 11, no. 14, pp. 1955-1961, 2017.

[4] P. Petrov, A. Radkovskaya, C. J. Stevens, and E. Shamonina, "Superdirectivity for coupled dimers of meta-atoms at mhz," in 2017 11th International Congress on Engineered Materials Platforms for Novel Wave Phenomena (Metamaterials), Aug 2017, pp. 265-267.

[5] M. Al-Nuaimi, "Compact size high gain lens corrected pyramidal horn antenna for 71-76 ghz band," 2014, pp. 23-25.

[6] HFSS, "Getting started with HFSS: Begineers guide," http://www.ansoft. com, vol. 1, July, 2016.

[7] C. Balanis, Antenna Theory. Wiley, ISBN:0-471-66782-X, 2005.

[8] H. Jasik, Antenna Engineering Handbook, 1st ed. MCGraw-Hill Book Company, 1961, chap. 16.

[9] A. Sihvola and J. Kong, "Effective permittivity of dielectric mixtures," IEEE Trans. on Geoscience and Remote Sensing, vol. 26, no. 4, pp. 420429, July 2000. 